\title{
Les disparitions politiques en RSA : des stratégies d'acteurs multiples
}

\section{Philippe Chapleau}

\section{(2) OpenEdition \\ 1 Journals}

\section{Édition électronique}

URL : http://journals.openedition.org/conflits/183

DOI : 10.4000/conflits.183

ISSN : $1777-5345$

Éditeur :

CCLS - Centre d'études sur les conflits lilberté et sécurité, L'Harmattan

Édition imprimée

Date de publication : 15 mai 1994

ISSN : 1157-996X

Référence électronique

Philippe Chapleau, «Les disparitions politiques en RSA : des stratégies d'acteurs multiples », Cultures \& Conflits [En ligne], 13-14 | printemps-été 1994, mis en ligne le 14 mars 2006, consulté le 30 mars 2021. URL : http://journals.openedition.org/conflits/183 ; DOI : https://doi.org/10.4000/conflits.183

Ce document a été généré automatiquement le 30 mars 2021.

Creative Commons License 


\title{
Les disparitions politiques en RSA : des stratégies d'acteurs multiples
}

\author{
Philippe Chapleau
}

1 Avec un record de 4.364 morts politiques en 1993, l'Afrique du Sud reste un pays hautement instable où la violence ne cesse de s'intensifier en dépit du démantèlement de l'apartheid. Depuis 1990, année charnière qui a vu la libération de Nelson Mandela et la suspension par le Congrès national africain (ANC) de la lutte armée, les chiffres annuels de la violence politique révèlent une augmentation constante du nombre des victimes et une exacerbation des tensions intra-communautaires. Des tensions qui s'expriment de plus en plus ouvertement au sein même de la communauté noire où les Zoulous - politiquement minoritaires mais tribalement majoritaires - mènent une politique d'agression et de confrontation.

2 Cette augmentation annuelle du chiffre des victimes tourne autour de $10 \%$. En 4 ans, alors que la libéralisation s'intensifiait, la violence politique a fait environ 10.000 morts. Un chiffre à peu près égal à celui des victimes de la répression - directe et indirecte menée par les autorités sud-africaines entre 1960 et 1989. Ces chiffres sont à manier avec précaution : ils ne signifient en rien que l'apartheid et sa cohorte de mesures coercitives étaient moins inducteurs d'instabilité que la libéralisation initiée par le président Frédérick de Klerk. Ils révèlent avant tout une polymorphie croissante de la conflictualité intra-étatique en Afrique du Sud et le passage d'une stratégie répressive menée par le pouvoir blanc pendant trois décennies à une stratégie agressive à laquelle ont dorénavant recours toutes les minorités qui évoluent sur la scène politique: extrême-droite blanche, forces de sécurité encore incapables d'en finir avec la répression musclée, activistes zoulous d'Inkatha, militants de la gauche noire et groupuscules africanistes.

3 La disparition politique: absence de recensement L'étude des statistiques communiquées annuellement par les différents groupes d'observation révèle également que la classification "disparitions politiques" est inexistante alors même que des centaines de milliers de personnes ont "disparu". Arrestations, détentions, blessés, morts, actions policières, assassinats par les escadrons de la mort ou les milices 
conservatrices : tout est répertorié, en particulier par la Human Rights Commission (la Commission sud-africaine des droits de l'homme). Mais il semble que les disparitions politiques ne constituent - et n'ont constitué selon eux- qu'un phénomène marginal ; les bulletins mensuels de la Human Rights Commission n'accordent d'ailleurs qu'une attention épisodique aux "disparitions". D'où une interrogation essentielle: les disparitions politiques ne constitueraient-elles qu'un phénomène négligeable dans un Etat hautement polémogène comme l'Afrique du Sud?

4 Les disparitions : stratégies des pouvoirs Pour se convaincre du contraire, il suffit de se reporter à l'ouvrage qu'un journaliste sud-africain a consacré aux escadrons de la mort. Dans "Death squads: Apartheid's secret weapon", Patrick Laurence cite les noms de nombreux disparus : pour la plupart des activistes enlevés dans les années 80 et dont le sort n'a été connu qu'après novembre 1989 , date à laquelle les premières confessions d'anciens membres des escadrons de la mort ont commencé à apporter quelques éclaircissements sur certaines disparitions. Laurence rapporte le cas de Siphiwe Mtimkulu, un jeune militant étudiant de Port-Elizabeth.

5 Arrêté en 1981, Mtimkulu a passé cinq mois au secret avant d'être libéré en octobre de la même année. Quelques jours après sa mise en liberté, il a commencé à souffrir de douleurs à l'abdomen, à perdre ses cheveux et à avoir d'énormes difficultés à marcher. A l'hôpital de Groote Schuur, au Cap, des examens ont révélé que Mtimkulu avait été empoisonné. Sa famille a alors déposé plainte contre le ministre de la police et demandé 150.000 rands de dommages et intérêts pour tentative de meurtre par empoisonnement. Peu après, Siphiwe Mtimkulu disparaissait. Un des camarades, Topsy Madaka, avec lui. On ne devait jamais les revoir.

6 Les cas cités par Laurence et d'autres observateurs des tactiques répressives sudafricaines montrent en fait que deux scénarios étaient envisageables dans les années 70 et 80 .

7 La disparition, dans le premier type de scénario, aboutissait au "suicide", comme ce fut le cas pour le syndicaliste blanc Neil Agget en 1982, ou à l'accident alors que le "disparu" était en détention: on se rappellera alors les troublants décès d'Imam Haran en 1969 et de Steve Biko en 1977. Entre septembre 1963 et juin 1990, 73 prisonniers sont officiellement morts en prison. Selon les magistrats, la moitié d'entre eux se seraient suicidés ; l'autre moitié auraient été victimes d'accident. Pendant cette même période, un seul policier a été condamné pour le meurtre d'un détenu.

8 Le second type de scénario mettait en scène des escadrons de la mort. En fait, ce second scénario a supplanté le premier au cours des années 80 . Suicides et accidents provoquaient à chaque occurrence une vague de protestations de la communauté internationale. La disparition physique permettait de résoudre toutes les difficultés : l'opposant était discrètement éliminé, son corps disparaissait. Ce qui laissait planer certains doutes sur le sort du disparu qui aurait très bien pu choisir la clandestinité par exemple. Les enlèvements étaient menés par les membres de diverses formations : par le CCB (Civil Cooperation Bureau) ou par les "contras" de la police sud-africaine basés à Vlakplaas, près de Pretoria.

9 Les cas de disparitions dues aux actions du pouvoir ont donc bien existé. Pourtant, l'opposition anti-apartheid n'en a jamais fait un cheval de bataille. Hommes d'église, syndicalistes, intellectuels et responsables du secteur associatif ont bien davantage dénoncé les arrestations massives et les assassinats que les disparitions d'opposants. 

certains aspects de la réalité quotidienne sud-africaine au cours des 20 dernières années.

Les disparitions: stratégies "sociétales" En fait, en un peu moins de 10 ans, des centaines de milliers de personnes ont disparu. La plupart temporairement, d'autres de façon plus fatale. Mais il existait, au sujet de tous ces disparus, une incertitude fondamentale qui ne permettait pas d'accuser formellement les autorités de conduire une politique suivie pour faire disparaitre les opposants et d'entretenir la terreur. Cette incertitude était fondée, puisqu'il existait plusieurs raisons convaincantes pour refuser d'assimiler systématiquement absence à disparition forcée. Il y a donc un intérêt des organisations à ne pas mettre l'accent sur le nombre de personnes disparues puisque nombre d'entre elles le faisaient volontairement.

12 Premier facteur : la législation. En 1984, interpellé par le député libérale Helen Suzman, le ministre de l'Intérieur d'alors annonçait qu'au cours des dix années passées, 637.584 Africains avaient été arrêtés pour défaut de production du Pass. Le Pass déterminait les droits de résidence et de passage des Noirs en zone blanche. Il avait été imposé en 1958 pour les hommes et en 1963 pour les femmes. Pour présence illégale dans une zone "européenne", un Noir pouvait être arrêté et emprisonné s'il ne disposait sur lui de la somme nécessaire pour payer l'amende. Avant que le président R.W. Botha, en janvier 1986, ne décide de supprimer le Pass, des milliers de Noirs ont ainsi disparu pendant quelques heures ou quelques mois, sans que leurs familles ne soient averties.

13 Pour aussi discriminatoire qu'elle soit, cette mesure régulant la présence des Noirs n'appartenait pas à l'arsenal législatif spécifique qui permettait au gouvernement blanc de réprimer toute velléité d'opposition de la part des communautés de couleurs et ainsi de prévenir toute activité politique. Pièces les plus lourdes de cet arsenal, le "Public safety act" et l'"Internal security act".

14 Le "Public safety act" (PSA) de 1953 permettait au chef de l'Etat de déclarer l'état d'urgence (comme en 1960, 1985, 1986, 1987, 1988, 1989) et conférait des pouvoirs illimités aux forces de l'ordre. Il permettait en outre d'imposer une censure quasitotale des médias. L'"Internal security act" (ISA) a été voté en 1982. Ses dispositions sont permanentes et donnent aux "sécurocrates" sud-africains le droit d'interdire des organisations, de censurer les individus, de prévenir les rassemblements et les manifestations, d'interdire certaines publications et, disposition des plus notables, d'emprisonner sans obligation de jugement toute personne pour 180 jours. La mise en détention est décidée sur simples présomptions d'activité séditieuses ou sur des "bases raisonnables" déterminées par n'importe quel policier ! Quant à la période de 180 jours, pendant laquelle le prévenu est au secret, elle peut pour le maintien de l'ordre public, être reconduite ad infinitum. Une fois de plus, et bien que des chiffres précis n'aient jamais été fournis par les autorités, on peut penser que plusieurs milliers de personnes ont ainsi "ponctuellement" disparu.

15 Autre facteur d'incertitude : l'entrée dans la clandestinité. Alors que les mouvements d'opposition étaient un à un déclarés illégaux, on a recensé, et cela surtout dans les années 80 , un nombre important de passages à la clandestinité. Activistes recherchés par la police, militants se sentant menacés : nombreux ont été les Sud-Africains qui ont choisi de se fondre dans la masse africaine des townships pour y poursuivre la lutte ou y survivre. Beaucoup ont vite appris que le silence et la confidentialité étaient de règle et que ni leur famille, ni leurs amis ne devaient connaitre leur nouvelle identité ou 
adresse. Ces "disparus" ont souvent refait surface en 1990, au moment où les premiers exilés rentraient de Zambie ou de Tanzanie.

Ces exilés, dont on estime le chiffre à 100.000, avaient choisi de fuir l'apartheid. Leur départ constitue un troisième facteur d'incertitude. Ainsi on a vu en 1976, après l'échec du mouvement lycéen et le début d'une répression impitoyable, des milliers de jeunes passer les frontières botswanaise ou zimbauwéenne pour tenter de rejoindre les rangs de l'ANC ou du PAC (le Congrès pan-africaniste). Motivé par la nécessité de se mettre à l'abri de poursuites pour les uns, ou la volonté de poursuivre le combat en choisissant la lutte armée pour d'autres, cet exil a conduit de nombreux jeunes Noirs à quitter leurs cercles de vie, souvent sans prévenir. D'où une fois encore des suspicions d'enlèvements. Mais déclarer une disparition présumée pouvait mettre la police sur une piste dont elle n'était pas consciente, lui permettre de découvrir le nom d'un opposant qui aurait gagné les rangs de l'opposition armée. Beaucoup de familles ont donc préféré taire ces absences : mieux valait l'incertitude que l'erreur, le silence que le mot malheureux qui risquait de faire marquer des points aux forces de l'ordre.

Ces trois facteurs d'incertitude ont en outre été exacerbés par l'extrême mobilité de la population noire et par les liens parfois très distendus qui prévalaient entre certains membres des cellules familiales ou tribales. L'exode rural a drainé des millions de Noirs vers les zones urbaines; les déplacements de population ont obligé plusieurs autres millions à quitter leurs zones de résidences habituelles pour s'installer dans les homelands ; ajoutons à cela une intense mobilité professionnelle et l'on comprendra qu'il était aisé de perdre de vue un parent, un ami, un voisin.

Mobilité des populations, exil vers les pays de la ligne du front anti-apartheid, entrée dans la clandestinité, emprisonnement : tous ces facteurs sont donc à bien considérer lorsqu'on veut aborder la question des disparitions politiques. Leur prise en compte ne constitue pas une façon d'occulter ce phénomène de violation des droits de l'homme mais elle permet de mieux mesurer l'ampleur et l'impact réel des disparitions en tant que stratégie de pouvoir sur la scène politique sud-africaine. Si l'on en croit les rapports récents le nombre des disparitions forcées a donc été réduit : moins de $5 \%$ du total des victimes politiques. Sans doute cette stratégie n'était-elle pas payante pour les autorités dans un pays où l'activisme politique ne se laissait pas arrêter même par les autres méthodes répressives.

\section{INDEX}

Mots-clés : disparitions

Index géographique : Afrique australe, Afrique du Sud (R.S.A.)

Thèmes : ANC 